\title{
BIM 技术在绿色公共建筑设计中的应用分析
}

\author{
杨荣洁 \\ 河北建筑设计研究院有限责任公司，河北 石家庄 050011
}

[摘要] 文章强调了 BIM 技术在绿色公共建筑设计中的应用优势, 并对 BIM 技术在绿色公共建筑设计中的具体应用进行了简单 分析。在此基础上, 以某绿色公共建筑项目为例, 从室外环境分析、室内环境分析、其他关键的绿色建筑这三方面入手, 阐 述了基于 BIM 技术的绿色公共建筑设计要点。 [关键词]BIM 技术; 绿色公共建筑; 建筑设计 DOI：10.33142/aem.v3i1.3654 中图分类号: TU242 文献标识码：A

\section{Application analysis of BIM Technology in Green Public Building Design}

YANG Rongjie

Hebei Institute of Architectural Design \& Research Co., Ltd., Shijiazhuang, Hebei, 050011, China

\begin{abstract}
This paper emphasizes the application advantages of BIM Technology in green public building design and analyzes the specific application of BIM Technology in green public building design. On this basis, taking a green public building project as an example and starting from the outdoor environment analysis, indoor environment analysis and other key green buildings, this paper expounds the key points of green public building design based on BIM Technology.
\end{abstract}

Keywords: BIM Technology; green public building; architectural design

引言

在当前的公共建筑设计中, 绿色建筑理念的融入受到重点关注, 此时, 需要最大程度利用自然采光、自然通风、 雨水等, 达到降低建筑物使用时的能源消耗的效果。出于对设计可操作性、合理性以及美观性的考量, 在绿色公共建 筑设计中应用 BIM 技术是必然选择，相应使用策略也值得重点探究。

\section{BIM 技术在绿色公共建筑设计中的应用优势分析}

在达到绿色设计、可持续性设计领域, BIM 的优点是很显著的：BIM 方法可用以分析包含影响绿色条件的采光、能 源效率和可持续性材料等建筑性能的各个方面; 可分析、达到最少的耗能, 并借助通风、采光、气流组织以及视觉对 人心理体会的控制等, 达到节能环保; 选用 BIM 理念, 还可在项目方案完成的同时, 测算日照、模拟风环境, 为建筑 设计的 “绿色探索” 注入高科技力量 ${ }^{[1]}$ 。综合来看, 在绿色公共建筑设计中应用 BIM 技术具有极高的现实价值。

\section{BIM 技术在绿色公共建筑设计中的具体应用分析}

第一, 节能体系的设计。为了满足绿色建筑要求, 必须要引入节能设计。实践中, 需要提前利用 BIM 软件完成建 筑物在不同条件下的实际能耗计算, 对比规定数值, 如果发现仿真分析中所获取到的能耗数值更大, 则必须要实施对 建筑节能体系的优化设计。

第二, 建筑物室外设计。在绿色建筑物的室外设计环节中, 主要利用 BIM 软件完成对采光情况的优化设计, 避免 光污染问题发生的同时优化室内的采光情况, 充分发挥出太阳能的作用。

第三, 建筑物室内设计。照明、通风等均为绿色建筑物室内设计环节需要重点考量的内容, 在 BIM 软件的支持下, 可以确定出最优的采光设计方案以及通风方案, 营造更为舒适的室内环境, 并最大程度降低能源消耗。

\section{3 基于 BIM 技术的绿色公共建筑设计实例分析}

\section{1 项目概述}

某公共建筑的总建筑面积为 $15590 \mathrm{~m}^{2}$, 为地上两层建筑, 建筑物高度达到 $12.6 \mathrm{~m}$ 。该公共建筑被规划为低碳体验馆, 主要以 “绿色低碳” 为理念完成设计, 并在其中融入生态景观、现代科技以及低碳技术, 属于三星级绿色建筑。在本 项目工程的设计过程中, 主要应用了 BIM 技术。

\section{2 室外环境分析}

(1) 室外风环境: 在 BIM 技术的支持下, 对该项目工程的建筑形体实施初步建模, 通过在 Revit 中导入双曲面屋 
顶, 并以此为基础完成建筑结构模型的搭建, 体现出将该建筑屋顶结构设计为随地形起伏的双曲面的表现。在 BIM 软 件中完成该建筑物的初始模型搭建后, 将导出的. sat 格式文件转变为 st1 格式文件, 为后续室外风环境的优化分析提 供支持。将提取到的 stl 格式文件上传至 Phoenics 软件内, 以此达到仿真模拟分析建筑物室外风环境的效果, 同时, 必须要参考该分析结果对建筑物的形体结构展开优化调整。

本公共建筑的预设建设长度为 $250 \mathrm{~m}$, 初步模拟计算的结果表明, 在该地域条件下, 如果在本公共建筑物的设计中 引入条形建筑结构的形式, 则产生建筑物周边风速突变区、局部死区的概率大幅提升, 无法切实保证该建筑物的室外 风环境始终稳定在良好的状态下。基于这样的情况, 为了进一步优化建筑外部行人区域空气流通情况, 主要结合建筑 物施工区域的地形引入了曲面形式完成建筑外观的设计。

（2）建筑表面风压：对于本建筑项目来说，其面对着湖泊、北侧覆土且在建筑物的南侧区域不存在高层建筑物的遮 挡。在夏季条件下, 东南风为施工区域的主导风向, 此时该建筑物需要迎面承受不低于 $2.2 \mathrm{~Pa}$ 的风压。该建筑物的高度 较低为, 因此室外风压的最低值发生在建筑物的顶部区域, 数值约为 $-1.6 \mathrm{~Pa}$ 。基于这一样的建筑表面风压分析结果, 在 实际的设计阶段可以在建筑物的顶部区域开设天窗结构, 保证建筑物的室内可以实现自然通风。在实际的设计过程中, 为了 规避死区、旋浴等问题的发生, 主要对整个建筑划分为三部分, 依托中间走廊完成连接。通过这一样的设计方式, 不仅可以 降低空气在建筑物室内的流动距离, 缩短空气在建筑物是室内的滞留时间长度, 达到优化建筑物室内自然通风的效果。

\section{3 室内环境分析}

(1)室内采光: 将在 BIM 软件中构建起的建筑结构初级模型以 gbxm1 格式文件的形式进行导出, 并在转移至 Ecotect 中后实施对建筑物室内环境的分析, 并以此为参考完成建筑物的室内采光设计 ${ }^{[2]}$ 。在本建筑物项目中, 主楼与配楼顶部 区域分别加设采光天窗, 在模型中对比不同形状、位置的采光天窗设计形式, 确定出最符合环境条件、设计要求的建 筑物室内采光设计方案。

(2) 室内通风: 依托在 BIM 软件中的分析能够了解到, 当建筑物室外温度为 $20^{\circ} \mathrm{C}$ 时, 受到室外温度低于室内温度 的影响, 在热压力驱动条件下, 建筑物室内的气流会向室外流动。当开启天窗后, 空气流速稳定在每秒 $0.5 \mathrm{~m}$ 的水平下, 室内空气龄最大值为 $2000 \mathrm{~s}$; 如果不设置天窗, 则室内空气龄最大值为 $2800 \mathrm{~s}$ 。对比结果表明, 通过设置可开启天窗, 空气在建筑物室内的滞留时间长度大幅缩短, 有效改善室内空气的质量。基于这样的情况, 在本次建筑物的室内通风 设计中，主要在展厅顶部区域加设可开启天窗结构，以此达促进建筑物室内自然通风的效果。

\section{4 其他关键的绿色建筑}

（1）地源热百系统: 本建筑项目为低碳体验馆, 所以在室内区域的中部位置规划为低碳展示区, 并将地热洜源作 为冷热源; 设置两台制冷量、制热量机组, 其中一台用于对热量进行回收并为生活热水提供热源支持。出于对优化展 示效果的考量, 主要将该地源热泉机房设置为供人参观的展厅。实践中, 主要利用 BIM 技术中包含的碰撞检测单元, 对该热源地沝机房的位置设置、其中包含的管线配置方式等等展开检测, 避免出现管道或空间碰撞等问题, 并提升管 线设计的有序性，强化地源热百机房设计质量的同时优化其空间美观程度。

(2) 雨水系统: 在本建筑项目中, 主要将屋面雨水采集与处理方式设计为虹吸雨水排水方式。实践中, 建筑物部 分屋面区域的初期雨水通过室外弃流池实现排出; 对于后期雨水, 则被转移至地下雨水设备房内包含的原水收集池中 进行保存, 方便后续对雨水的集中处理及再利用; 对于剩余的建筑屋面雨水, 经过管线直接排放至式户雨水管网内, 最终汇入市政雨水系统。选用渗水砖进行建筑物室外场地、停车场的地面铺装, 以此达到提升雨水下渗量的效果, 降 低地表径流系数以及雨水地表径流量, 规避地面热岛效应的发生。

（3）太阳能光伏发电：对于本建筑物项目而言，其南方拥有良好的日照与采光条件，实践中，主要在日照环境条 件以及绿色建筑要求的指导下, 结合 BIM 技术完成太阳能光伏发电的设计, 即在建筑物的南面加设了外遮阳系统 (两 侧)。针对建筑物中部区域的低碳展示区, 引入了自动外遮阳系统 (在太阳高度变化后发生改变), 结合太阳能光伏发 电板的集成应用, 确保所设置的立面太阳板可以获取到太阳照射的最大值。

\section{4 总结}

综上所述，在绿色公共建筑设计中应用 BIM 技术具有极高的现实价值。通过在绿色公共建筑物节能体系、建筑室 内以及室外的设计引入 BIM 技术, 结合对室外风环境、建筑表面风压、室内采光、室内通风、雨水系统、太阳能光伏 发电的优化设计, 提升了绿色公共建筑设计的效率效果。

\section{[参考文献]}

[1]吉喆,徐飞. BIM 技术在绿色公共建筑设计中的应用分析 [J]. 工程建设与设计, 2020(7): 173-174.

[2]白佳程. BIM 技术在绿色公共建筑设计中的应用研究 [J]. 智能建筑与智慧城市, 2019 (12) : 63-64.

作者简介: 杨荣洁 (1977.9-) 女, 毕业院校: 石家庄铁道学院; 现就职单位: 河北建筑设计研究院有限责公司。 